\section{BMJ Paediatrics Open}

\title{
Pilot study of an integrated model of sleep support for children: a before and after evaluation
}

Heather E Elphick (D) , ${ }^{1}$ Candi Lawson, ${ }^{2}$ Ann Ives, ${ }^{2}$ Sue Siddall, ${ }^{2}$ Ruth N Kingshott, ${ }^{1}$ Janine Reynolds, ${ }^{1}$ Victoria Dawson, ${ }^{3}$ Lorraine Hall ${ }^{2}$

To cite: Elphick HE, Lawson C, Ives $\mathrm{A}$, et al. Pilot study of an integrated model of sleep support for children: a before and after evaluation. BMJ Paediatrics Open 2019;3:e000551. doi:10.1136/ bmjpo-2019-000551

- Additional material is published online only. To view please visit the journal online (http://dx.doi.org/10.1136/ bmjpo-2019-000551).

Received 8 July 2019 Revised 2 October 2019 Accepted 12 October 2019

\section{Check for updates}

(C) Author(s) (or their employer(s)) 2019. Re-use permitted under CC BY-NC. No commercial re-use. See rights and permissions. Published by BMJ.

${ }^{1}$ Department of Respiratory and Sleep Medicine, Sheffield Children's Hospital, Sheffield, UK ${ }^{2}$ Sheffield City Council, Sheffield, UK

${ }^{3}$ The Children's Sleep Charity, Doncaster, UK

Correspondence to Heather E Elphick; h.elphick@ nhs.net

\section{ABSTRACT}

Objective Despite the success of behavioural sleep support interventions in the third sector, sleep support is not universally available for families in the UK. The aim of the study was to provide evidence of efficacy and to propose a delivery model for integrated sleep support for families of vulnerable children.

Design and setting A sleep support intervention was carried out in Sheffield Local Authority evaluated using a preintervention and postintervention study design by Sheffield Children's National Health Service (NHS) Trust. Participants Fifty-six children aged 6-16 years with significant sleep problems were recruited; 39 completed the intervention and evaluation.

Interventions Basic sleep education and an individualised programme was delivered by a sleep practitioner. Follow-on telephone support was provided to empower the parent (and/or young person) to carry out the sleep programme at home. An integrated NHS and Local Authority delivery model was designed and implemented. Results Parents' ratings of their child's ability to selfsettle improved from $1.1 / 10$ to $6.4 / 10(p<0.05)$. Mean Warwick-Edinburgh Mental Well-being Scale scores improved significantly for parents/carers (MD 5.16, $95 \%$ Cls 2.62 to $7.69, p<0.05)$. Children who completed the intervention gained on average an extra 2.4 hours sleep a night. There was reduction in healthcare utilisation, illnesses and medication use.

Conclusions The behavioural approach to sleep support for these vulnerable groups of children is highly effective. Follow-on individual support to empower parents is key to achieving success. Sleep support can be implemented in NHS and Local Authority services by integration into the existing workforce using a cross-agency model.

\section{INTRODUCTION}

Sleep is a restorative process, fundamental to physical and psychological health. ${ }^{1}$ Approximately $30 \%$ of young children experience sleep difficulties in the form of bedtime resistance and night time waking; termed as behavioural insomnia. ${ }^{2}$ These difficulties can arise from the way parents manage their child's sleep $^{3}$ and can be effectively treated with a behavioural approach. ${ }^{4}$

The impact of sleep disturbance on children's health is wide-ranging including

\section{What is known about the subject?}

Sleep deprivation due to behavioural insomnia has an impact on physical, mental and emotional health and well-being for the child and family.

- Intensive sleep support interventions are effective but access to support is patchy and, in most areas, offered only by the voluntary sector.

- Integrated multiagency working is a National Health Service priority area.

\section{What this study adds?}

Cross-agency sleep support delivered via an integrated delivery model has shown efficacy and can be implemented by integration into the existing workforce.

- Using this approach, an average of an extra 2.4 hours of sleep per night was achieved as well as a significant improvement in parent/carer well-being.

- Key to achieving success with the intervention was the use of follow-up motivational telephone support

difficulties with mood, psychosocial problems and a detrimental impact on the child's cognitive ability and learning. ${ }^{56}$ Parents of children with sleep difficulties can suffer high levels of stress and anxiety, decreased ability to work or to drive safely, relationship and financial problems. ${ }^{7-9}$ These stresses lead to an increased demand on National Health Service (NHS) primary care services and to prescriptions of drugs such as antidepressants.

Evidence from sleep clinics delivered in the voluntary sector has shown that an intensive behavioural intervention can be highly effective. ${ }^{10}$ However, recognition of sleep deprivation as a factor in psychosocial morbidity has not been an NHS priority and resources are scarce.

A partnership comprising Sheffield Children's NHS Trust (SCH), Sheffield City Council and The Children's Sleep Charity (TCSC) carried out and evaluated an intensive 
behavioural intervention delivered by community practitioners to provide support to parent/carers and young people to improve sleep patterns for vulnerable children (The Sheffield Children and Young People Sleeping Well Project). A proposed integrated delivery model was developed and has begun to be implemented to provide cross-agency sleep support within the city.

\section{METHODS}

The study design was an observational pre-evaluation and postevaluation of a behavioural sleep intervention in the form of a workshop or clinic appointment with follow-up support. A control group was not included.

\section{Patient and public involvement}

Members of the Sheffield parent carer forum were involved in the concept and development of the study. Two parent/carers sat on the strategic committee which discussed strategies for gathering evidence and implementation of city-wide services.

\section{Recruitment}

Children and young people aged 6-16 years known to have a sleep problem and with either with attentiondeficit hyperactivity disorder (ADHD) or looked after/ adopted children (LAAC) were selected as being two groups of children highlighted by service managers as being vulnerable and therefore priority groups for intervention. Participants were selected sequentially on referral from an ADHD clinician or key worker dealing with LAAC who felt that the child/family would benefit from the intervention. Participants were not known to sleep practitioners or the research team before referral to the project and only had contact for the duration of the project. Children with a Composite Sleep Disturbance Index ${ }^{11}$ score of 3 or more, indicating significant problems with settling to sleep and/or waking at night were eligible for inclusion. Inclusion criteria were checked by the research team prior to recruitment. Children with a specific physiological sleep or medical disorder (eg, sleep apnoea, pain, respiratory or gastrointestinal symptoms or seizures) were excluded if it was felt that the sleep disturbance had a medical basis that should be prioritised over the sleep support intervention. This was screened for by the practitioners at initial assessment and discussed with the consultant (HEE) as necessary. HEE made the final decision as to whether or not the child was included. Children were also excluded if there were factors such as clinical (physical or mental health) or social life-events that would have interfered with the implementation of the sleep intervention within the time-frame of the project. We therefore did include children with other sleep problems such as sleep walking if it was considered that the child may still benefit from the sleep support intervention with no other concerns identified. Those that did not proceed with the study were signposted back into the appropriate clinical or Local Authority services for further evaluation.

\section{Intervention}

Two experienced practitioners with Parenting and Learning Mentor roles within the Local Authority early intervention team delivered the sleep support interventions over a 10-month period. The practitioners had previously received accredited sleep practitioner training through TCSC (www.thechildrenssleepcharity.org.uk). The objective of the intervention was to provide bespoke support to parents/carers and/or young people, to skill and empower them to implement a behavioural sleep programme at home to improve their child's/their nighttime behaviour with ongoing support from the practitioners.

The intervention was delivered via a 3-hour workshop for four to six families or via a 1:1 clinic model (decided on a case-by-case basis according to age, preference and workshop availability). In both settings, education about basic sleep physiology was given followed by a one-to-one consultation to explore possible solutions to sleep problems by developing an individualised sleep programme. The programme included a consistent routine, removal of technology from the bedroom, hand-eye co-ordination activities for the hour before bedtime, melatoninproducing supper-time foods, avoidance of caffeine and changes to the bedroom environment.

The delivery was targeted at parents in the case of children aged 1-11 years and at the young person with or without parents in the case of young people aged 12-15 years. To engage teenagers in the process, a clinic appointment was offered for them to attend with their parent/carer. The session was designed around talking to the young person, finding out the difficulties from their perspective, talking through their needs and the barriers to good sleep, with reference to some basic sleep science. The teenagers were empowered to develop their own sleep programme with the practitioner supporting them to devise their own solutions. A bespoke programme with a generic sleep information pack was given to each individual participant.

\section{Primary goal}

Parents were asked to set a goal for the intervention outcome, for example to settle more quickly or to sleep through the night without wakening. Parents selected these themselves. Some parents had two or more goals. The parents were asked to pick a score from a number 1-10 on a chart for each of their goals (figure 1: Goal progress chart) and this score was recorded at baseline and at every contact throughout the intervention period. The Goal progress chart was used to demonstrate improvement in order to motivate and reassure the parents. Following conclusion of the intervention, the parents' self-selected scores at baseline and at conclusion were compared. 
Name

GOAL:

\begin{tabular}{|c|c|c|c|c|c|c|c|c|c|c|c|c|}
\hline Session & \multirow[t]{2}{*}{ Date } & \multicolumn{11}{|c|}{$\begin{array}{l}\text { Today I would rate progress to this goal: } \\
\text { (Please circle the appropriate number below) }\end{array}$} \\
\hline \multicolumn{12}{|c|}{$\begin{array}{c}\text { Remember a score of zero means no progress has been made towards a goal, a score or ten means a goal has been } \\
\text { reached fully, and a score of five is exactly half way between the two. }\end{array}$} & \\
\hline 1 & & 0 & 1 & 2 & 3 & 4 & 5 & 6 & 7 & 8 & 9 & 10 \\
\hline 2 & & 0 & 1 & 2 & 3 & 4 & 5 & 6 & 7 & 8 & 9 & 10 \\
\hline 3 & & 0 & 1 & 2 & 3 & 4 & 5 & 6 & 7 & 8 & 9 & 10 \\
\hline 4 & & 0 & 1 & 2 & 3 & 4 & 5 & 6 & 7 & 8 & 9 & 10 \\
\hline 5 & & 0 & 1 & 2 & 3 & 4 & 5 & 6 & 7 & 8 & 9 & 10 \\
\hline 6 & & 0 & 1 & 2 & 3 & 4 & 5 & 6 & 7 & 8 & 9 & 10 \\
\hline 7 & & 0 & 1 & 2 & 3 & 4 & 5 & 6 & 7 & 8 & 9 & 10 \\
\hline 8 & & 0 & 1 & 2 & 3 & 4 & 5 & 6 & 7 & 8 & 9 & 10 \\
\hline 9 & & 0 & 1 & 2 & 3 & 4 & 5 & 6 & 7 & 8 & 9 & 10 \\
\hline 10 & & 0 & 1 & 2 & 3 & 4 & 5 & 6 & 7 & 8 & 9 & 10 \\
\hline 11 & & 0 & 1 & 2 & 3 & 4 & 5 & 6 & 7 & 8 & 9 & 10 \\
\hline 12 & & 0 & 1 & 2 & 3 & 4 & 5 & 6 & 7 & 8 & 9 & 10 \\
\hline
\end{tabular}

(1) Sheffield City Council

Figure 1 Goal progress chart. Parent/carers were asked to suggest a goal to work towards and to score their impression of their current achievement of that goal at each contact with the sleep practitioner by circling their chosen number on the chart. The progress documented on the chart served as a motivational tool.

\section{Follow-up support}

Follow-up support for parents and young people was in the form of telephone calls, texts or email contact according to the individual's preference, for as long as was needed to achieve the parent/young person's goal or to reach a level that was considered to be a successful or unsuccessful intervention by the parent/young person and practitioner. This was not predefined but was the point at which no further input from the practitioner was deemed beneficial, that is, the primary goal score was no longer improving.

\section{Evaluation}

An independent research team carried out home visits at baseline and 1 month following the sleep intervention programme to measure the impact of the sleep problems on the child and family, and to obtain written informed consent.

Outcome measures evaluated were as follows:

- Strength and Difficulties Questionnaire (SDQ) for parent/carer, teacher and self-assessment (children aged $11-15$ years).

- Warwick-Edinburgh Mental Well-being Scale to measure impact on parent/carer mental well-being. The scale measures 14 aspects of positive mental health including feeling relaxed, thinking clearly, feeling confident and cheerful.

- Self-designed questionnaires to measure:

- the child's sleep pattern, based on parental recollection, including time taken to settle, time taken to fall to sleep, number of nights per week the child woke in the night, number of per night, duration of nocturnal wakenings and total sleep duration per night (online supplementary file 1 ).

- parent/carer well-being - a rating of the impact on quality of life in the previous 2 weeks of a series of factors rated 1-5 (online supplementary file 2 ).

- days missed from school; days missed from work in the previous 2 weeks.

- health of parents and child and visits to healthcare and non-healthcare professionals (HCP) in the previous 2 weeks.

Feedback on the intervention itself (including negative feedback) was also assessed at the follow-up evaluation using a self-designed questionnaire.

\section{Statistics}

A sample size calculation was not carried out due to the pilot study design. The recruitment numbers were dependent on the number of workshops and clinics feasible to provide during the funded study period of 10 months.

Participants who withdrew from the study were taken into account in the analysis by imputing values for the final outcome measure carried forward from the baseline evaluation. In addition, a per protocol analysis of the patients who completed the intervention and evaluation was carried out. For comparison of scores from baseline to postintervention, mean differences, SD, 95\% CIs and two-tailed independent $\mathrm{t}$-tests were calculated using Excel.

\section{Development of implementation model}

The proposed implementation model was developed through a strategic group which included the core team, 


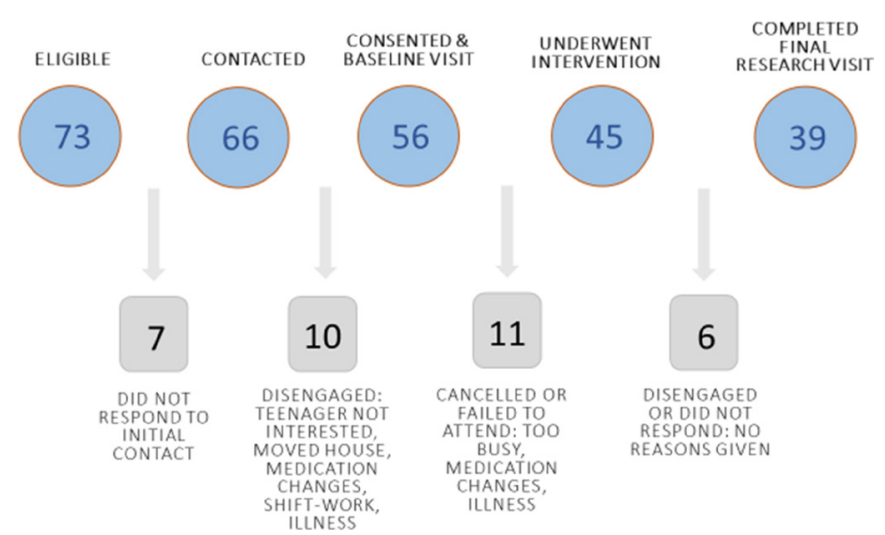

Figure 2 Project flowchart. Thirty-four participants either did not respond to initial contact from the sleep practitioners or disengaged. Ten participants failed to attend or cancelled the workshop or clinic appointments after the baseline visit. After completing the workshop or clinic, one family did not respond to contacts for arranging the final visit and five families disengaged without giving a reason. Thirty-nine participants completed the final evaluation.

service managers from the 0 to 19 health service and Local Authority parenting service, medical and pharmacy advisors from Sheffield CCG, SCH Trust, Looked After and adoption services, the Sheffield parent carer forum and Children's Residential Homes. The findings and proposed model were presented to the Children's Health and Well-being Transformation Board, the Executive Director of People Services Portfolio (Children's and Adults) and the elected member for Children and Young People.

Both NHS and Local Authority ethics approvals and a formal data sharing agreement were obtained (REC reference 16/YH/0490).

\section{RESULTS}

Seventy-three eligible participants were approached. Seventeen of these potential participants were identified by front-line staff as eligible but did not want to engage with the project. Reasons given for not consenting were as follows: young person did not wish to take part, moved house recently, recent medication changes meant sleep no longer an issue, illness, parent working shifts or no reason given.

Fifty-six patients were recruited to the study (median age 8.7 years; range $1.8-15.7$ years; 45 males: 11 females). Forty-two had ADHD, seven were adopted, four lived in residential homes and three were in foster care. Figure 2 outlines the reasons for withdrawal from the study. The overall withdrawal rate was $30 \%$. Eleven participants completed the baseline evaluation but did not attend the sleep support intervention. Reasons given were as follows: too busy, could not travel into the city centre for the intervention, recent medication changes meant sleep no longer an issue, severe escalation of the child's mental health problems. All 45 participants who started the intervention completed it (including telephone support). Six participants dropped out between completion of the intervention and the final evaluation. Reasons for this were given as carer unable to continue with the bedtime routine (1) or not given (5). Ten out of the 17 families that withdrew had a lone parent. Thirty-nine participants completed the final evaluation (median age 8.6 years; range $1.8-15.7$ years; 31 males: 8 females). Of those that completed, 18 attended a workshop and 21 attended a 1:1 clinic. Twelve of this group had a lone parent; 29 children had ADHD, five were adopted, three lived in residential homes and two were in foster care.

\section{Primary goal}

Primary goal scores were collected from all 45 families that received the intervention. The goals self-selected by parents were grouped into the following themes: to sleep through the night without wakening $(\mathrm{n}=4)$; to self-settle $(n=10)$; to fall asleep more quickly $(n=28)$; stay in their own bed $(n=2)$; to feel less tired in the day $(n=1)$. The median initial score (out of 10) for the parents' primary goal was 0 (range 0-6); the median final score was 7.5 (range 0-10). The mean initial score was 1.1; the mean final score was 6.4. The change in mean goal score was statistically significant (MD 4.10, 95\% CIs 3.75 to 4.42, $\mathrm{p}<0.05)$.

\section{Strengths and difficulties questionnaires}

Fifty-five parents completed the SDQ at baseline, 37 teachers and eight young people. With missing data imputed from baseline data, none of the measures were significantly changed following the intervention. (Results included in online supplementary material.)

\section{Warwick-Edinburgh mental well-being scale}

For the overall study group $(\mathrm{n}=56)$ with scores imputed from baseline scores for the participants who withdrew from the study, the mean score improved significantly following the intervention from 39.5 to 44.6 (MD 5.16, $95 \%$ CI 2.62 to $7.69, \mathrm{p}<0.05)$. For the 39 who completed the intervention and evaluation, the mean score improved significantly following the intervention from 39.0 to 46.8 (MD 8.84, 95\% CI 5.32 to 12.36 , $\mathrm{p}<0.05$ ).

\section{Sleep questionnaire}

Table 1 shows the results of the sleep questionnaire. There was no difference in the time taken to fall asleep in the participants who completed compared with those that withdrew from the study (mean time 2.1 hours both groups) or the total sleep time (6.27 hours in the completed group and 6.21 hours in the withdrawals group). All but one of the mean changes from baseline to postintervention were statistically significant $(\mathrm{p}<0.05)$. Of those that completed, the average number of extra hours sleep per night was 2.4 hours (range 0.5 hours less to 7.5 hours more sleep per night). Taking into account withdrawals prior to and following the intervention and imputing baseline scores into the outcome measures, the average number of extra hours sleep was calculated as 1.63 hours. 
Table 1 Results of the sleep questionnaire. all participants $(n=56)$

\begin{tabular}{|c|c|c|c|}
\hline Variable & Baseline (mean) & $\begin{array}{l}\text { Postintervention } \\
\text { (mean) }\end{array}$ & Mean difference (MD); 95\% Cl \\
\hline \multicolumn{4}{|l|}{ All participants $(n=56)$} \\
\hline Time to settle (hours) & 2.03 & 1.37 & MD $0.67 ; 95 \% \mathrm{Cl} 0.25$ to $1.08, \mathrm{p}<0.05$ \\
\hline Time to fall asleep (hours) & 2.1 & 1.1 & MD $0.99 ; 95 \% \mathrm{Cl} 0.61$ to $1.38, \mathrm{p}<0.05$ \\
\hline Number of nights wake per week & 4.3 & 2.8 & MD 1.52; $95 \% \mathrm{Cl} 0.71$ to $2.32, \mathrm{p}<0.05$ \\
\hline Number of wakenings per night & 1.8 & 1.09 & MD $0.68 ; 95 \% \mathrm{Cl} 0.28$ to $1.08, p<0.05(n=54)$ \\
\hline Duration of wakenings (min) & 49.51 & 29.05 & $\begin{array}{l}\text { MD 14.98; } 95 \% \mathrm{Cl}-6.11 \text { to } 36.08, p=0.18 \\
(n=41)\end{array}$ \\
\hline Number of hours sleep (hours) & 6.25 & 7.88 & MD $1.63 ; 95 \% \mathrm{Cl} 1.04$ to $2.23, \mathrm{p}<0.05$ \\
\hline \multicolumn{4}{|l|}{ Participants who completed (n=39) } \\
\hline Time to settle (hours) & 1.76 & 0.81 & MD $0.95 ; 95 \% \mathrm{Cl} 0.5$ to $1.4, \mathrm{p}<0.05$ \\
\hline Time to fall asleep (hours) & 2.1 & 0.67 & MD 1.43; $95 \% \mathrm{Cl} 0.95$ to $1.91, \mathrm{p}<0.05$ \\
\hline Number of nights wake per week & 4.0 & 1.87 & MD 2.18; $95 \% \mathrm{Cl} 0.55$ to $2.36, \mathrm{p}<0.05$ \\
\hline Number of wakenings per night & 1.54 & 0.58 & MD $0.97 ; 95 \% \mathrm{Cl} 0.43$ to $1.39, \mathrm{p}<0.05$ \\
\hline Duration of wakenings (min) & 33.59 & 10.55 & MD 23.31; $95 \% \mathrm{Cl} 7.65$ to $35.6, \mathrm{p}<0.05$ \\
\hline Number of hours sleep (hours) & 6.27 & 8.62 & MD 2.35; 95\% Cl 1.64 to $3.06, p<0.05$ \\
\hline
\end{tabular}

\section{Parent/carer well-being}

When asked about the impacts on parental well-being of the child's sleep problem, 50/56 primary respondents were the mother, $3 / 56$ were care-workers and $3 / 56$ were foster carers. All well-being scores improved following the intervention and all but two reached statistical significance (figure 3 ).

Days missed from school reduced from 20.9 to 18.5; number of days missed from work reduced from 9.2 to 0 . Number of visits to HCP and non-HCP reduced by 4 and 23, respectively. There were fewer reported illnesses in parents/carers (headaches, anxiety, depression and general tiredness) and in children (viral illnesses and colds). None of these measures of the wider impacts of the sleep intervention reached statistical significance but all showed improvement.

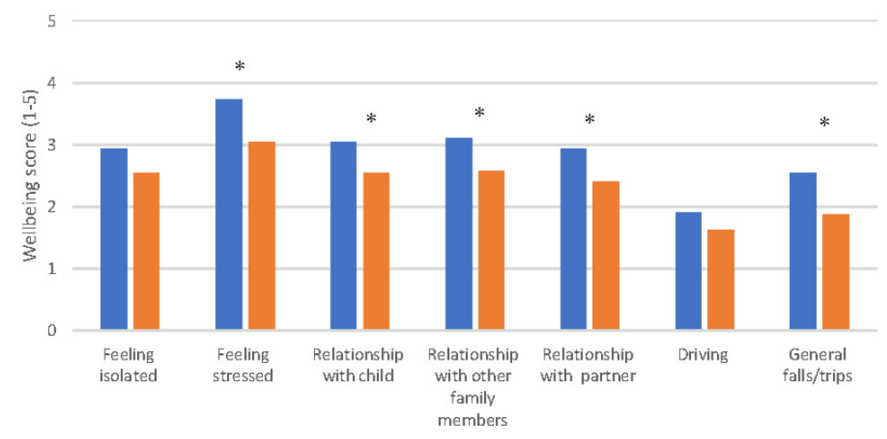

Figure 3 Parent/carer well-being scores preintervention and postintervention. scores were on a scale of $1-5$, with one being none of the time and five being all of the time, that is, a low score indicated good quality of life and a high score indicated poor quality of life. ${ }^{*} p<0.05$.

\section{Parent/career feedback}

Of the 39 parent/carers who responded following the intervention, 31 felt the intervention had helped their child, 33 reported that the intervention had helped their role as a parent or carer and 32 believed the intervention helped other children in the household.

Some parents felt that behaviour was unchanged and that the child still could not switch off at bedtime. However, the majority of comments at the final visit were positive, noting improvements at school, "no more battles', the ability to start new activities and go on holiday, improved energy, confidence and relationships. Of note, $100 \%$ parents/carers said that they would recommend the programme, even if it had not been successful for their child. The key enabling factor in the parental feedback was the regular telephone support.

\section{Implementation model}

The implementation model (figure 4) was developed using a whole systems/whole population approach looking at complexity of need against breadth of reach, ranging from awareness raising and promotion, through universal settings, targeted support for complex situations to specialist support. The final step in the referral pathway is the Sheffield Children's Hospital Clinic.

Where sleep interventions are delivered, the model is mainly based on a hub and spoke model, with existing staff taking on sleep as part of their role and Sleep Practitioner leads (either geographically or within specific service areas) driving and supporting implementation.

By building capacity into the workforce, members of staff have been trained as sleep practitioners across the key services: Parenting, Health visiting, Inclusion, SEND, Children's Residential Homes. All early help staff have 


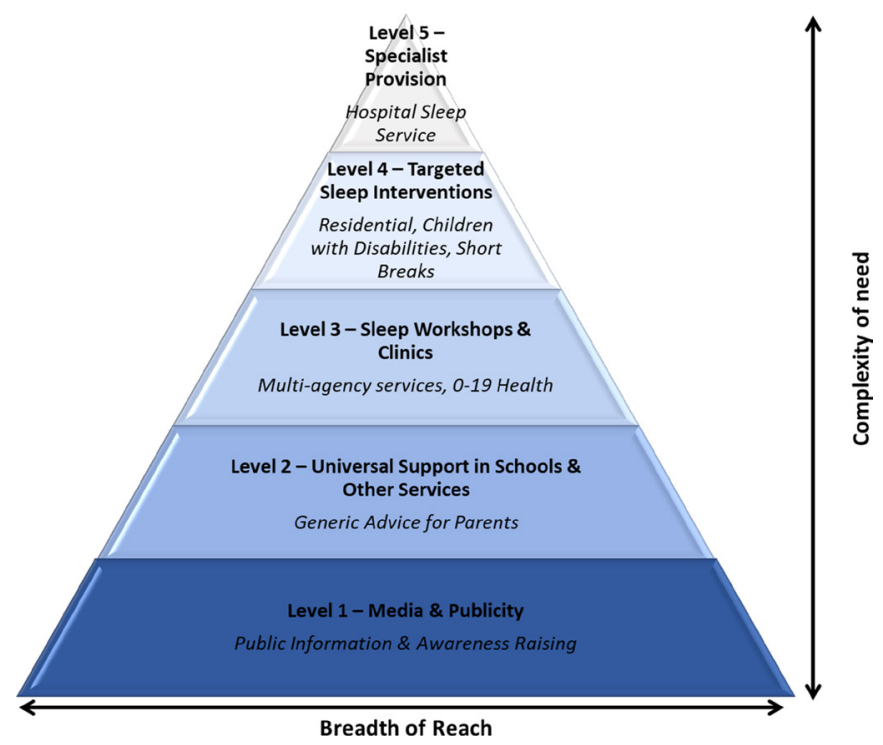

Figure 4 The proposed implementation model for crossagency delivery of sleep support services.

been given awareness training and staff trained in 24 schools.

\section{DISCUSSION}

The key findings from this evaluation were that, with an individual sleep programme and support from a sleep practitioner, children's total sleep time and parent/carer mental well-being were significantly improved. In addition, parents' confidence in their ability to achieve their goals, healthcare utilisation, illnesses and medication use were also improved. Through partnership working a local integrated model of sleep support delivery has been established.

The finding that a behavioural intervention for sleep support is effective is not novel. Previous projects have shown efficacy of behavioural sleep support delivered both by the third sector and in healthcare settings. ${ }^{12-14}$ Our study has shown that delivery and implementation of sleep support within existing local services is feasible for groups of vulnerable children in the community. We have shown that empowering parents to implement consistent sleep routines at home, giving them a sense of achievement, as well as more sleep, led to a significant improvement in mental well-being. The support offered by the practitioners is a complex package of care using a whole systems approach. This package has not been formally described in terms of a behaviour change model and future research to explore this further would be beneficial to understanding the most effective elements of the intervention package. Our observation was that the effectiveness of the 1:1 clinic model and the group workshop were equivalent, but that parent's preferences differed. The key aspect of the delivery model that parents consistently reported to be the most effective was the follow-up support in the form of phone calls or emails.
Barriers to the implementation of the intervention to families included engagement of the young person (usually around negotiating removal of technology), finding the optimum time at which to introduce the programme around other events at home or other therapies taking priority, parental tiredness and mental state and logistics such as other children in the home or lack of support for the parent. Parental motivation was another factor as many felt they had tried sleep support before or believed that other issues such as the ADHD diagnosis would prevent the intervention from being helpful. The skill needed to motivate parents and young people beyond their initial beliefs is a requirement of a sleep practitioner as well as a knowledge of sleep. A consistent and whole household approach is crucial, along with appropriate timing.

Recruitment was opportunistic and relied on an initial approach and referral to the project by a member of the child's clinical team (for ADHD patients) or key worker (for LAAC). The recruitment of participants was therefore dictated by the referral rate possible within the timeframe of the project. Time was devoted at the beginning of the project to visit the appropriate agencies with information about the project and recruitment process. Barriers to recruitment were largely centred around the availability and engagement of the referring staff with some expressing a great deal of enthusiasm for the project and others citing lack of time and changes in management structure as barriers to engagement.

There was a $30 \%$ withdrawal rate from our study with $11 / 56$ participants unable to proceed with the intervention. Despite this, all participants who started the intervention did complete it and only $6 / 56$ were lost to follow-up. The baseline characteristics of the children whose parent/carer withdrew were the same as those that completed the intervention and evaluation in terms of gender, age, underlying diagnosis or residential placement. The only difference noted was that $10 / 17$ families that withdrew had a lone parent and 12/39 of the group that completed had a lone parent. This factor may have impaired their ability to engage and should be highlighted for particular support in future implementation of this intervention. Reasons for withdrawal cited by the families were generally practical, including house moves, mental illness and changes to medication. In a real-life setting, these factors would have been noted and support offered to parents at a later date, however, given the time constraints of a research study, it was not possible to include these families further in this project.

Caution is needed when interpreting the results of this observational study. Without a concurrent control group, it cannot be assumed that observed changes were directly due to the intervention. While the changes may have been influenced by other interventions introduced at the time of the study, it is likely however that the sleep intervention did have significant results in these children with severe long-term sleep problems. Time points were not standardised and varied from patient to patient 
depending on the complexity of the case and the number of contacts needed for each individual family. It was therefore decided that, rather than analysing according to non-standardised time points, that only the preevaluation and postevaluation time points would be analysed. We accept that parental observation of children's sleep times is subjective and may be inaccurate. However, in asking the same parent to assess their child before and after the intervention it was deemed to be as accurate as possible in the absence of an objective measure. Our experience of objective measurements of sleep duration such as actigraphy is that children's sleep is disturbed by the presence of the monitor and although this method was considered, it was not feasible within the scope of the study and therefore subjective measures were used.

Due to the withdrawal rate, two analyses were carried out. A per protocol analysis was carried out on those participants who completed the intervention and provided data on the outcome measures. We recognise however that this analysis results in an over-representation and represents the maximum potential or 'best case' results. Analysis taking into account withdrawals was therefore also carried out based on the assumption that baseline data would remain unchanged without the intervention and using imputed values for the final outcome measure by carrying forward the baseline value for research participants lost to follow-up. These results represent the "worst case' and an under-representation of the outcomes because the 11 participants who had received the intervention and were likely to have improved from baseline. In reality, the outcomes that truly reflect the intervention are likely to lie between those generated by the two analysis methods.

One of the enabling factors for the project was the partnership between the Children's Hospital Trust, the City Council and TCSC. The integrated working across local authority, health and third sector brought together providers and commissioners with expertise in strategic planning, research and sleep. The Sleep Practitioners' experience in parenting and education enabled them to understand complex situations over and above the sleep difficulties which were fundamental to addressing some of the complex situations that arose. Challenges faced by the joint initiative included the requirement to submit the project to both NHS and local authority governance frameworks. Aligning this process and different organisational cultures and expectations was more challenging than anticipated. However, the partnership working opened doors and opportunities that otherwise would not be available, ultimately leading to better provision for families.

The proposed implementation model was designed to address the concepts of whole family working to connect parenting, health and well-being with sleep and to create accessible support for all families. Barriers to the implementation of the service model were twofold-workforce and training resources and engagement of services and individual staff. However, since oral dissemination of the results in our region has taken place some of these barriers are being overcome. There remain a number of resource gaps which include continued workforce training, support networks, awareness raising and signposting to services and resources, expansion into other services including primary care, co-ordination and triage, implementation and mentorship materials for school, accessible information online and further evidence gathering and research.

We suggest that this relatively inexpensive approach can be implemented in both local authority and health services by integration into the existing workforce. We would recommend that staff training in sleep support and early intervention using an integrated cross-agency model would benefit children and their parents and save NHS healthcare costs by addressing issues as a first-line intervention before escalation to more specialist services.

Acknowledgements We would like to thank the children and their families and carers for taking part in the project. We would also like to thank the Ryegate Children's centre, CAMHS, MAST and SEND services for their support throughout the project.

Contributors Contributorship Statement. All authors were involved in the planning and reporting of the study, all authors have reviewed the final manuscript. HEE led the study, supervised the research team, led the NHS strategic planning, analysed the results and wrote the main body of the report. CL and LH led the Local Authority strategic planning and supervised the sleep practitioners. SS and Al delivered the sleep practitioner intervention. VD trained and supervised the sleep practitioners and led the charity strategic planning. RNK and JR delivered the evaluations, data collection and recording.

Funding This work was supported by The Health Foundation grant ref 3042. Competing interests None declared.

Patient consent for publication Not required.

Provenance and peer review Not commissioned; externally peer reviewed.

Data availability statement Data are available upon reasonable request.

Open access This is an open access article distributed in accordance with the Creative Commons Attribution Non Commercial (CC BY-NC 4.0) license, which permits others to distribute, remix, adapt, build upon this work non-commercially, and license their derivative works on different terms, provided the original work is properly cited, appropriate credit is given, any changes made indicated, and the use is non-commercial. See: http://creativecommons.org/licenses/by-nc/4.0/.

\section{ORCID iD}

Heather E Elphick http://orcid.org/0000-0001-8982-9250

\section{REFERENCES}

1 Walker M. Why we sleep: the new science of sleep and dreams ISBN-13: 978-0241269060. 1st edn. Allen Lane, 2018.

2 American Academy of Sleep Medicine. International Classification of Sleep Disorders. In: Diagnostic and coding manual. 2nd edn. Westchester, IL, 2005.

3 Wiggs L. Behavioural aspects of children's sleep. Arch Dis Child 2009;94:59-62.

4 Meltzer LJ. Clinical management of behavioral insomnia of childhood: treatment of bedtime problems and night wakings in young children. Behav Sleep Med 2010;8:172-89.

5 Simola P, Liukkonen K, Pitkäranta A, et al. Psychosocial and somatic outcomes of sleep problems in children: a 4-year follow-up study. Child Care Health Dev 2014;40:60-7.

6 Dewald JF, Meijer AM, Oort FJ, et al. The influence of sleep quality, sleep duration and sleepiness on school performance in children and adolescents: a meta-analytic review. Sleep Med Rev 2010;14:179-89.

7 Montgomery P, Wiggs L. Definitions of sleeplessness in children with attention-deficit hyperactivity disorder (ADHD): implications 
for mothers' mental state, daytime sleepiness and sleep-related cognitions. Child Care Health Dev 2015;41:139-46.

8 Doo S, Wing YK. Sleep problems of children with pervasive developmental disorders: correlation with parental stress. Dev Med Child Neurol 2006;48:650-5.

9 Tietze A, Zernikow B, Michel E, et al. Sleep disturbances in children, adolescents, and young adults with severe psychomotor impairment: impact on parental quality of life and sleep Developmental Medicine \& Child. Neurology 2014;56:1187-93.

10 Turner K, Bullock L, Down A, et al. Sleep tight: an evaluation of a community based intervention on the sleep behaviours of young children. Journal of Health Visiting 2016;4:572-8.

11 Quine L. Sleep problems in children with mental handicap. J Mental Defic Res 1991;35:269-90.
12 Scantlebury A, Mcdaid C, Dawson V, et al. Non-Pharmacological interventions for non-respiratory sleep disturbance in children with neurodisabilities: a systematic review. Dev Med Child Neurol 2018;60:1076-92.

13 Keshavarzi Z, Bajoghli H, Mohamadi MR, et al. In a randomized case-control trial with 10-years olds suffering from attention deficit/ hyperactivity disorder (ADHD) sleep and psychological functioning improved during a 12-week sleep-training program. The World Journal of Biological Psychiatry 2014;15:609-19.

14 Corkum P, Lingley-Pottie P, Davidson F, et al. Better nights/better days-distance intervention for insomnia in school-aged children with/without ADHD: a randomized controlled trial. J Pediatr Psychol 2016;41:701-13. 